\title{
Factors Associated with Physical Activity
}

\author{
Armend Kastrati ${ }^{1}$ and Georgi Georgiev ${ }^{2}$ \\ 'University of Pristina "Hasan Pristina", Pristina, Kosovo, ${ }^{2}$ Ss. Cyril and Methodius University, Faculty of Physical Education, Sport, and Health, \\ Skopje, Republic of North Macedonia
}

\begin{abstract}
The purpose of this paper is to determine the association of demographic, psychological, and social factors with physical activity, and to determine whether indicators of physical activity differ in terms of gender among 974 young adults of Albanian nationality from 18 to 29 years of age. Students completed a questionnaire that examined their level of participation in physical activity and behaviours, along with several potential correlates. Hierarchical regression was used to explore the relationship between hypothesised factors and physical activity. On the basis of the obtained results, one can conclude that male students, unlike female students, show a significantly higher level of physical activity. The respondents of both genders who have a higher level of self-efficacy perceive greater benefits of physical activity, greater social support from friends, and come from families where a larger number of family members have higher levels of physical activity. The results of the research suggest the importance of the preparation of a national plan and programme to promote physical activity to help young people change unhealthy lifestyle habits and increase physical activity, thereby to improve their health.
\end{abstract}

Keywords: physical activity, students, attitudes, IPAQ criteria

\section{Introduction}

The influence of physical activity (PA) on human health has been proven in numerous studies but, unfortunately, a large part of the population remains insufficiently physically active (Hallal, Victora, Azevedo, \& Wells, 2006; Bouchard, Blair, \& Haskell, 2007; WHO, 2010). Diseases caused by hypokinesis constitute a significant problem in modern medicine. Sports and recreational physical activities are an indispensable means for compensating the lack of movement. For this reason, many developed countries around the world have created state-wide strategies to promote physical activity among their citizens, which is a priority in the attempt to reduce the massive healthcare costs in the long run.

In adolescents, the health benefits of physical activity include healthy growth and development of the bones as well as the muscular and cardio-respiratory system, maintenance of energy balance, avoiding risk factors for cardiovascular diseases, possibilities for social interaction, and positive mental well-being: higher self-esteem, lower anxiety, and lower stress
(Hillsdon \& Foster, 2003; British Heart Foundation, 2004).

Given the fact that as age increases, physical activity decreases, adolescence is a critical period of intervention (Aaron et al., 1993). Adolescents are more likely to be physically active in adulthood if they establish habits to engage in physical activity in early adolescence (Telama \& Yang, 2000). Physical activity is a complex behaviour that is affected by many internal and external factors, including socio-cultural factors, psychological-cognitive factors, and the physical and social environments that surround the individual.

The explanation regarding the influence of the factors on behaviour changes is crucial in designing interventions, strategies and educational programmes that will contribute to increasing the level of physical activity in young people (Sallis, Prochaska, \& Taylor, 2000). Current guidelines recommend that all young people should participate in physical activity of at least moderate intensity for one hour per day.

The results of the previous research indicate that self-efficacy (confidence in one's abilities to perform a particular

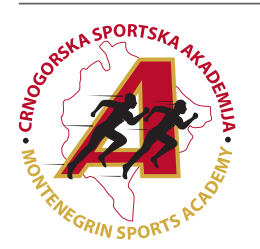

Correspondence:

G. S. Georgiev

Ss. Cyril and Methodius University, Faculty of Physical Education, Sport, and Health, Str. Dimche Mirchev 3, 1000 Skopje, Republic of North Macedonia

E-mail: georgigeorgiev63@yahoo.com 
behaviour in specific situations), the increase of the level of perceived benefits of physical activity, the reduction of the perceived barriers, the increase of social support from the closest persons in the environment (parents and peers/friends), the enjoyment in physical activity, and the access to equipment and facilities are factors associated with physical activity in the period of adolescence (Sallis et al., 2002; Sallis et al., 2000).

In the Republic of Kosovo, there is a small number of studies that investigated factors that affect physical activity in adolescents who have their own socio-cultural, religious, and other specific factors. In contrast, internationally this topic has been the subject of numerous studies in many countries and different geographical environments; however, the question arises of whether the results of these studies can be generalized to the population of Albanian adolescents (McKenzie et al., 2002; Schmitz et al., 2002). In response, this study has been conducted in order (a) to determine the association of demographic, psychological and social factors with physical activity, (b) to determine whether physical activity indicators differ in terms of gender, (c) and to provide recommendations for interventions aimed at promoting an active and healthy lifestyle in this population group.

\section{Methods}

\section{Participants}

The research was conducted on a sample of 974 randomly selected respondents from several faculties within the university. The sample consisted of 572 (58.7\%) male respondents (male students) and 402 (41.3\%) female respondents (female students). The average age of the respondents of both genders was 20.2 years. The respondents were treated according to the Declaration of Helsinki.

\section{Measures}

A questionnaire was used to identify (a) demographic factors, (b) the physical activity, (c) the factors associated with physical activity.

\section{Demographic Factors}

A ten-item investigator-developed questionnaire was used to collect demographic data. The following demographic data and participant characteristics were collected to describe the study sample: age, gender, residence, number of family members, education of the father, education of the mother, whether the father is active in sport and whether the mother is active in sport.

\section{Physical Activity}

The nine-item IPAQ-C records self-reported physical activity in the last seven days. Responses were converted to Metabolic Equivalent Task minutes per week (METmin/wk) according to the IPAQ scoring protocol: total minutes over previous seven days engaged in vigorous activity, moderate-intensity activity, and walking were multiplied by $8.0,4.0$, and 3.3 , respectively, to create MET scores for each activity level. MET scores across the three sub-components were totalled to indicate overall physical activity.

\section{Exercise Benefits/Barriers Scale}

The original version of EBBS consisted of 43 items, 29 items under the construct of benefits, and 14 items under the construct of barriers. The benefit scales are composed of five subscales: life enhancement, physical performance, psychological outlook, social interaction, and preventative health.
The barrier scales are composed of four subscales: exercise milieu, time expenditure, physical exertion and family discouragement. The scales are designed based on a four-point Likert scale: strongly agree (4), agree (3), disagree (2), strongly disagree (1). When the barriers and benefits are added together for a total benefits/barriers score, the items of the barrier scale have an inversed score. The barrier items are not inversely scored if only the barrier items are used. The benefit subscales can have a score ranging from 29 to 116 and the barrier subscales can have a score ranging from 14 to 56. Altogether, they result in a total score between 43 and 172. In this scoring system, the higher score represents the greater perception of a subscale.

\section{Exercise Self-Efficacy}

Perceived exercise self-efficacy was assessed using the Children's Physical Activity Self-Efficacy Survey, an eightitem scale developed by Garcia, Pender, Antonakos, and Ronis (1998). Items included, for example, "I could exercise even if I were tired" and "I could exercise even if I were not very good at it". Responses ranged from "very true" to "not at all true" and a mean score, ranging from $1-5$, was computed by averaging responses to the eight items.

\section{Parental Support}

Levels of paternal and maternal support for being active were measured using an adapted version of a scale from the Amherst Health and Activity Study (Sallis et al., 2002). Five items assessed different aspects of parental support, including encouragement, participation in joint activities, provision of transport, observation and praise. Responses ranged from "a lot" to "not at all". A mean score, ranging from 1-5, was computed by averaging responses to the five items.

\section{Peer Support}

Peer support for being active was measured using an abridged version of the parental support scales. Two items assessed how often friends provided encouragement to be active and how often they took part in joint activities. Responses ranged from "a lot" to "not at all". A mean score, ranging from $1-5$, was computed by averaging responses to the five items. The question "How much do you enjoy doing physical activity?" was asked, as well as asking respondents about their enjoyment of physical education at school and how many of their friends are physically active (Inchley, Kirby, \& Currie, 2008).

\section{Enjoyment}

The enjoyment of PA was assessed using the revised PACES, which was originally designed to measure positive effects associated with involvement in physical activities in college students (Kendzierski \& DeCarlo, 1991). It has since demonstrated internal consistency in 12-16 year-old children, with coefficient $\alpha=.90$, and item-total correlations $=.38-.76$ (Crocker, Bailey, Faulkner, Kowalski, \& McGrath 1997). The original PACES consisted of 18 bipolar statements on a seven-point continuum (I enjoy it-I hate it) which were totalled to produce a total enjoyment score. The revised PACES consists of 16 statements that begin with the stem "When I am physically active.... The items of the PACES questionnaire can be seen in Table 1. Motl, Dishman, Saunders, Dowda, and Pate (2007) modified the PACES for use with young adolescent females. In doing so, two items were removed and others rewritten to im- 
prove comprehension and reduce redundancy, and a five-point Likert-type scale (1="Disagree a lot" to 5="Agree a lot") which was considered more comprehensible to younger children replaced the 7-item bipolar continuum (Motl et al., 2007). A score is computed by calculating the average of the 16 items.

\section{Statistical Analysis}

The data was processed using the statistical package SPSS for Windows Version 22.0. The normality of the distribution of the variables was checked with the Kolmogorov-Smirnov method, and log transformations were made when possible. The variables that met the normality criterion were analysed by using parametric statistical procedures, while the variables that did not meet the normality criterion were analysed by using nonparametric statistical procedures. Independent samples t-tests and Mann-Whitney $\mathrm{U}$ tests were used to compare differences in PA for gender. Comparison of means used a two- tailed hypothesis with the alpha levels set at $\mathrm{p}<.05$. Spearman's Rho correlation was used to analyse the relationship between PA and potential correlates. Factors associated with PA were entered into hierarchical regression models.

\section{Results}

To determine whether there are differences in physical activity and psychosocial factors between male and female respondents, a Mann-Whitney U test was applied. From the overview of Table 1, which presents the results of the applied analysis, it is observable that statistically significant differences were found between male and female respondents in the variables: vigorous physical activity $(\mathrm{p}=0.000)$, moderate physical activity $(\mathrm{p}=0.000)$, total physical activity $(\mathrm{p}=0.000)$, exercise self-efficacy $(\mathrm{p}=0.000)$, peer support $(\mathrm{p}=0.001)$, perceived barriers of physical activity $(\mathrm{p}=0.003)$, perceived benefits of physical activity $(\mathrm{p}=0.004)$, and enjoyment of physical activity $(\mathrm{p}=0.001)$.

Table 1. Means and standard deviations for study variables grouped by gender and Mann Whitney U tests

\begin{tabular}{lccccc}
\hline \multirow{2}{*}{ Variables } & \multicolumn{2}{c}{ Males } & \multicolumn{2}{c}{ Females } & \multirow{2}{*}{ p } \\
\cline { 2 - 5 } & Mean & SD & Mean & SD & 0.000 \\
Vigorous & 1731.39 & 2042.26 & 694.79 & 1435.47 & 0.000 \\
Moderate & 770.22 & 1040.54 & 496.46 & 902.83 & 0.000 \\
Total FA & 4194.65 & 3164.46 & 2907.50 & 2627.60 & 0.000 \\
Self-Efficacy & 3.01 & 0.77 & 2.81 & 0.72 & 0.334 \\
Parent Support & 3.20 & 1.13 & 3.14 & 1.12 & 0.001 \\
Peer Support & 3.73 & 1.07 & 3.47 & 1.19 & 0.003 \\
Barriers & 31.94 & 6.80 & 30.64 & 6.30 & 0.004 \\
Benefits & 92.32 & 11.66 & 90.49 & 10.73 & 0.477 \\
Total (Benefit/Barrier) & 130.48 & 14.40 & 129.88 & 13.54 & 0.001 \\
Enjoyment & 3.25 & 0.35 & 3.19 & 0.33 &
\end{tabular}

Table 2 shows the coefficients of correlation between physical activity and demographic, social, and psychological variables in respondents of both genders. The Table 2 overview indicates that there is a low statistically significant positive correlation (with values within the range from .15 to .23) between physical activity and the treated variables: whether the father is active in sport, exercise self-efficacy, parent support, peer support, perceived benefits of physical activity and total score (Benefit/Barrier scale). For female respondents, there was a low and statistically significant positive correlation (with values within the range of .16 to .24 ) between physical activity and the variables: exercise self-efficacy, peer support, perceived benefits of physical activity and total score (benefit/ barrier scale), while a low negative correlation was found between physical activity and the variable of perceived barriers of physical activity.

Table 2. Correlations between Physical Activity and various factors using Spearman's Rho

\begin{tabular}{lcc}
\hline Variables & Males & Females \\
\hline Number of Family Members & .056 & .071 \\
Education of the father & -.112 & -.027 \\
Education of the mother & -.114 & -.067 \\
Whether the father is dealing with sport & $.151^{* *}$ & .006 \\
Whether the mother is dealing with sport & 0.05 & .072 \\
Self-Efficacy & $.228^{* *}$ & $.202^{* *}$ \\
Parent Support & $.129^{* *}$ & .092 \\
Peer Support & $.151^{* *}$ & $.162^{* *}$ \\
Barriers & -.084 & $-.152^{* *}$ \\
Benefits & $.225^{* *}$ & $.213^{* *}$ \\
Total (Benefit/Barrier) & $.213^{* *}$ & $.242^{* *}$ \\
Enjoyment & .096 & .047 \\
\hline
\end{tabular}


To determine the multivariate influence of the demographic, psychological, and social factors, a hierarchical regression analysis was applied. The results of the regression analysis are presented in Table 3. The first regression equation explains $8.4 \%(\mathrm{p}<0.001)$ of the total variability of the criterion variable, with statistically significant influence of predictor variables: gender, number of family members, father's education and whether the father was engaged in sports. By including the two social variables in the second step, the amount of the explained variance increases to $10.3 \%(\mathrm{p}<0.001)$, and the linear correlation coefficients in the equation for this system of 10 predictors with the physical activity level is $\mathrm{R}=0.321(\mathrm{~F}=10.30$, $\mathrm{p}<0.001)$. Social support from friends $(\beta=3.841, \mathrm{p}<0.001)$ is a significant determinant that has a significant statistical effect on the criterion variable physical activity. With the inclusion of the third block of psychological variables, the amount of the explained variable increases to $15.5 \%(\mathrm{p}<0.001)$. Of the entire predictive system, the variables of exercise self-efficacy $(\beta=5.183, p<0.001)$ and total score (Benefit/Barrier scale), $(\beta=4.350, p<0.001)$ have significant statistical effects. After including the third block of psychological variables, it is observable that the variable education of the father in the final regression equation becomes statistically insignificant. On the basis of the obtained results, it can be concluded that the education of the father is not directly related to the physical activity in this population group.

Table 3. Hierarchical regression analyses explaining PA

\begin{tabular}{|c|c|c|c|}
\hline Variables & B & Partial $\mathbf{r}$ & $B_{1}$ \\
\hline Demographics & \multicolumn{2}{|c|}{$\mathbf{R}=0.290 \mathbf{R}^{2}=0.084 * *$} & \\
\hline Age & -0.107 & -0.003 & -0.347 \\
\hline Gender & $-7.091^{* * *}$ & -0.223 & $-6.140^{* * *}$ \\
\hline Residence & -0.431 & -0.014 & -0.138 \\
\hline Number of Family Members & $3.272^{* * *}$ & 0.105 & $3.517^{* * * *}$ \\
\hline Education of the father & $-2.279^{*}$ & -0.073 & -1.836 \\
\hline Education of the mother & -0.626 & -0.020 & -0.393 \\
\hline Whether the father is dealing with sport & $3.223 * * *$ & 0.103 & $2.653^{* *}$ \\
\hline Whether the mother is dealing with sport & 1.855 & 0.060 & 1.541 \\
\hline Social & \multicolumn{2}{|c|}{$\mathbf{R}=0.321 \mathbf{R}^{2}=0.103 * * *$} & \\
\hline Parents support & 0.934 & 0.030 & -0.021 \\
\hline Peer support & $3.841^{* * *}$ & 0.123 & $2.019^{*}$ \\
\hline Psychological & \multicolumn{2}{|c|}{$\mathbf{R}=0.393 \mathbf{R}^{2}=0.155^{* * *}$} & \\
\hline Self-Efficacy & $5.183^{* * *}$ & 0.246 & $5.183^{* * *}$ \\
\hline Total (Benefit/Barrier) & $4.350 * * *$ & 0.212 & $4.350 * * *$ \\
\hline Enjoyment & 0.835 & 0.067 & 0.835 \\
\hline
\end{tabular}

Legend: Partial $r$ - coefficient of partial correlation; $\beta$ - standardized partial regression coefficient; $ß 1$ - the value of the $ß$-coefficients follows the following equation of analysis; $\mathrm{R}$ - coefficient of multiple correlation; $\mathrm{R}^{2}$ - change of the coefficient of determination; Significance, ${ }^{*} p<.05,{ }^{* *} p<.01,{ }^{* * *} p<.001$.

\section{Discussion}

Several previous studies suggest a decline in physical activity during adolescence, with a further decline in early adulthood. However, less information is available regarding what constitutes these changes and patterns of determinants for population subgroups. The understanding of the factors that contribute to decreased physical activity in adolescents is essential for developing effective programmes and strategies to promote physical activity in this population group.

Most researches to date suggest that the level of physical activity of girls is lower than that of boys (Higgins, Gaul, Gibbons, \& Van Gyn, 2003; Muntner et al., 2005; Wu \& Jwo, 2005; Raudsepp, 2006; Shi et al., 2006; Sherrick-Escamilla, 2007). The results of our research also indicate that male students show higher levels of physical activity with high and medium intensity and overall physical activity compared to female students. Moreover, boys also perceive greater levels of self-efficacy, greater benefits of physical activity, more reduced barriers from physical activity, greater social support from friends, and more enjoyment in physical activity compared to girls.
The social environment where the physical activity takes place has a crucial impact on young people. Family members, friends, teachers and coaches can play a significant role in promoting adolescent physical activity. The social influence can function through a variety of mechanisms, including encouragement, activity modelling, common activity, and practical support. The results of our research have shown that social support from parents does not have a significant effect on physical activity in this sample of respondents. Nevertheless, previous research suggests that the role of parents in promoting physical activity in their children is particularly important; most probably, it is more significant in younger respondents.

Other family characteristics, such as the socioeconomic status and the education of parents, may affect physical activity in adolescents. The results of the studies on the impact of the socioeconomic status on physical activity in children and adolescents are contradictory. The research carried out within the Scottish Health Survey suggests that girls from families with lower socioeconomic status have higher levels of physical activity than girls from families with higher socioeconomic status do. In contrast, the results of the research within the Health 
Behaviour of School-aged Children (HBSC) study suggest that adolescents from wealthier families are more physically active (Levin et al., 2007) and more likely to participate in vigorous exercise in their free time (Inchley et al., 2008).

In this study, a statistically significant association was identified between the number of family members and the sporting tradition in the family (the parent as a role model). The results from the Framingham Heart Study indicate that children of active parents are nearly six times more likely to be physically active than children of inactive parents (Moore et al. 1991).

Furthermore, social support from friends, and especially group activity (number of friends who exercise), is an important determinant that can help increase physical activity in young people. This has been confirmed in many previous studies conducted in children, preadolescents, and adolescents (Zakarian, Hovell, Hofstetter, Sallis, \& Keating, 1994).

The belief in the benefit of physical activity is also a significant predictor of physical activity among respondents of both genders, which has been confirmed in several studies involving preadolescents and adolescents (Zakarian et al., 1994).

Self-efficacy is a belief in one's abilities in attempts to accomplish a task. Self-efficacy rests on the idea about the importance of the subjective experience of personal competence in the achievement of different goals, rather than real knowledge, abilities and skills. Self-efficacy is not estimated as a personality trait, but rather as a belief in one's abilities to coordinate knowledge and abilities to achieve the desired goal. Personal factors (cognitive, emotional, and biological), environmental factors and behaviour have an interactive influence on the model of reciprocal determinism (Bandura, 1997). The results of this study show that self-efficacy, which is a construct of the social cognitive theory, is positively related to physical activity in respondents of both genders. The results of other studies also show that self-efficacy, directly and indirectly, affects physical activity in children and adolescents (Wu, 1999; Neumark-Sztainer, Story, Hannan, Tharp, \& Rex, 2003; Wu \& Pender, 2003; Chang, 2004; Wu \& Jwo, 2005; Jago, Baranowski, Baranowski, Cullen, \& Thompson, 2007; Motl et al., 2007; Sherrick-Escamilla, 2007).

Based on all the above-mentioned factors, it can be concluded that a national plan and programme for promoting

\section{Acknowledgements}

There are no acknowledgements.

\section{Conflict of Interest}

The authors declare that there are no conflicts of interest.

Received: 19 November 2019 | Accepted: 27 December 2019 | Published: 01 February 2020

\section{Reference}

Aaron, D.J., Kriska, A.M., Dearwater, S.R., Anderson, R.L., Olsen, T.D., Cauley, J.A., \& Laporte, R.E. (1993). The epidemiology of leisure physical activity in an adolescent population. Medicine and Science in Sports and Exercise, 25, 847-853.

Bandura, A. (1997). Self-efficacy: The exercise of control. New York: Freeman.

British Heart Foundation (2004). Couch kids: the continuing epidemic. London: British Heart Foundation

Chang, M.C. (2004). Behavioral and sociocultural influences on PA among Asian-American youth. Unpublished doctoral dissertation. Michigan, USA: University of Michigan, Ann Arbor.

Crocker, P.R.E., Bailey, D.A., Faulkner, R.A., Kowalski, K.C., \& McGrath, R. (1997). Measuring general levels of physical activity: preliminary evidence for the Physical Activity Questionnaire for Older Children. Medicine \& Science in Sports \& Exercise, 29(10), 1344-1349. physical activity need to be developed to help adolescents change unhealthy lifestyle habits and increase physical activity, and thus improve their health. These strategies, plans and programmes should be prepared by considering the specifics of the environment, the customs and the cultural characteristics of the region.

There is evidence that anyone who increases their level of physical activity can have health benefits even after a long period of inactivity, regardless of age.

Changes can be made through extensive modification in policy and practice, and in particular through an increase of the cross-sectoral cooperation and the adoption of new roles by different entities that are already proven and respected in their fields. Minor changes in policy and practice are needed, aimed to promote and increase physical activity among the young population.

Different approaches can be used: individual work, group work, workshops, counselling, among others. The primary promoter of these educational programs and strategies should be the school; however, it is necessary to involve more governmental and non-governmental organizations, the family as well as the local government and the country with a broad media campaign. Changes in the school should be directed towards changing the curricula that should include contents for physical activity and its importance, the inclusion of new forms of physical activity in the curriculum, improvement of the material base (facilities, devices, etc.).

Based on the obtained results, it can be concluded that boys show significantly higher levels of physical activity compared to girls. The respondents of both genders who have a higher level of self-efficacy perceive greater benefits of physical activity, greater social support from friends; they come from families in which more family members have higher levels of physical activity.

This study did not find a significant relation between the age of parents, the education of parents and physical activity. The sporting tradition in the family (the father as a role model) affects the physical activity of the respondents of both genders. The results of the research indicate the importance of the preparation of a national plan and programme to promote physical activity to help young people change unhealthy lifestyle habits and increase physical activity, thereby to improve their health.

Garcia, A.W., Pender, N.J., Antonakos, C.L., \& Ronis, D.L. (1998). Changes in Physical Activity Beliefs and Behaviors of Boys and Girls across the Transition to Junior High School. Journal of Adolescent Health, 22, 394402.

Hallal, P., Victora, C., Azevedo, R., \& Wells, J. (2006). Adolescent physical activity and health. A systematic review. Sports Med, 36, 1019-1030.

Higgins, J.W., Gaul, C., Gibbons, S., \& Van Gyn, G. (2003). Factors influencing physical activity levels among Canadian youth. Canadian Journal of Public Health, 94, 45-51.

Hillsdon, M., \& Foster, C. (2003). The relationship between physical activity during childhood and adolescence and coronary heart disease risk factors in young adulthood. In A. Giles (Ed.), A Lifecourse Approach to Coronary Heart Disease Prevention: Scientific and policy review (199-212). London: The Stationary Office.

Inchley, J., Kirby, J., \& Currie, C. (2008). Physical Activity in Scottish Schoolchildren (PASS) Project: Physical activity among adolescents in Scotland: final report of the PASS study. Child and Adolescent Health Research Unit. Edinburgh: The University of Edinburgh.

Jago, R., Baranowski, T., Baranowski, J.C., Cullen, K.W., \& Thompson, D.I. (2007). Social desirability is associated with some physical activity, psychosocial variables and sedentary behavior but not self-reported physical activity among adolescent males. Health Education Research, $22,438-449$.

Kendzierski, D., \& DeCarlo, J.K. (1991). Physical Activity Enjoyment Scale: Two 
validation studies. Journal of Sport \& Exercise Psychology, 13(1), 50-64.

Levin, K., Todd, J., Inchley, J., Currie, D., Smith, R., \& Currie, C. (2007). Family affluence and health among schoolchildren. HBSC Briefing Paper 11. Edinburgh: Child and Adolescent Health Research Unit. Edinburgh: University of Edinburgh.

McKenzie, T.L., Sallis, J.F., Broyles, S.L., Zive, M.M., Nader, P.R., Berry, C.C., et al. (2002). Childhood movement skills: Predictors of physical activity in Anglo American and Mexican American adolescents? Research Quarterly for Exercise and Sport, 73, 238-244.

Moore, L.L., Lombardi, D.A., White, M.J., Campbell, J.L., Oliveria, S.A., \& Ellison, R.C. (1991). Influence of parents' physical activity levels on activity levels of young children. J Pediatr, 118, 215-219.

Motl, R.W., Dishman, R.K., Saunders, R.P., Dowda, M., \& Pate, R.R. (2007) Perceptions of physical and social environment variables and selfefficacy as correlates of self-reported physical activity among adolescent girls. Journal of Pediatric Psychology, 32, 6-12.

Muntner, P., Gu, D., Wildman, R. P., Chen, J., Qan, W., Whelton, P. K., \& He, J. (2005). Prevalence of Physical Activity among Chinese Adults: Results from the International Collaborative Study of Cardiovascular Disease in Asia. American Journal of Public Health, 95(9), 1631-6.

Neumark-Sztainer, D., Story, M., Hannan, P.J., Tharp, T., \& Rex, J. (2003) Factors associated with changes in physical activity. A cohort study of inactive adolescent girls. Archives of Pediatric and Adolescent Medicine, $157,803-810$

Raudsepp, L. (2006). The relationship between socioeconomic status, parental support and adolescent physical activity. Acta Paediatrica, 95 93-98.

Sallis, J.F., Prochaska, J.J., \& Taylor, W.C. (2000). A review of correlates of physical activity of children and adolescents. Medicine \& Science in Sports \& Exercise, 32, 963-975.

Sallis, J.F., Taylor, W.C., Dowda, M., Freedson, P.S., \& Pate, R.R. (2002).
Correlates of vigorous physical activity for children in grades 1 through 12: comparing parent-reported and objectively monitored physical activity. Pediatric Exercise Science, 14, 30-44.

Schmitz, K.H., Lytle, L.A., Phillips, G.A., Murray, D.M., Birnbaum, A.S., \& Kubik M.Y. (2002). Psychosocial correlates of physical activity and sedentary leisure habits in young adolescents: the Teens Eating for Energy and Nutrition at School study. Preventive Medicine, 34, 266-278.

Sherrick-Escamilla, S. (2007). Factors affecting self-reported physical activity in children aged 10-12. Unpublished doctoral dissertation. Detroit, Michigan, USA: Wayne State University.

Shi, Z., Lien, N., Kumar, B.N., \& Holmboe-Ottesen, G. (2006). Physical activity and associated sociodemographic factors among school adolescents in Jiangsu province, China. Preventive Medicine, 43, 218-221.

Telama, R., \& Yang, X. (2000). Decline of physical activity from youth to young adulthood in Finland. Medicine and Science in Sports and Exercise, 32, 1617-1622.

WHO (2010). Global Recommendations on Physical Activity for Health. Geneva: World Health Organization.

Wu, T.Y. (1999). Determinants of physical activity among Taiwanese adolescents: An application of the health promotion model. Unpublished doctoral dissertation. Michigan, USA: University of Michigan, Ann Arbor.

Wu, T.Y., \& Jwo, J.L. (2005). A prospective study on changes of cognitions, interpersonal influences, and physical activity in Taiwanese youth. Research Quarterly for Exercise and Sport, 76, 1-10.

Wu, T.Y., \& Pender, N.J. (2003). Gender differences in the psychosocial and cognitive correlates of physical activity among Taiwanese adolescents: A structural equation modeling approach. International Journal of Behavioral Medicine, 10, 93-105.

Zakarian, J.M., Hovell, M.F., Hofstetter, C.R., Sallis, J.F., \& Keating, K.J. (1994). Correlates of vigorous exercise in a predominantly low socio-economic status and minority high school population. Preventive Medicine, 23(3), 31 